\title{
The Effects of Recency and Numerical Uncertainty Estimates on Overcautiousness ${ }^{\mathscr{D}}$
}

\author{
RAONI DEMNITZ AND SUSAN JOSLYN \\ University of Washington, Seattle, Washington
}

(Manuscript received 11 September 2019, in final form 14 January 2020)

\begin{abstract}
The four experiments reported here tested the impact of recent negative events on decision-making. Participants were given a virtual budget to spend on crops of varying costs and payoffs that, in some cases, depended on drought conditions. Participants made 46 decisions based on either deterministic or probabilistic seasonal climate predictions. Participants experienced a sequence of droughts either immediately prior to the target trials (recent condition) or early in the sequence (distant condition). In experiment 1, participants made overly cautious crop choices when droughts were experienced recently. Subsequent experiments probed the cognitive mechanisms involved. The effect of recency on overcautiousness was reduced by a midexperiment message, although it did not matter whether the message described a changed or consistent venue and time period. This suggests that overcautiousness was not caused by deducing a climatic trend in the particular area. Instead, we argue that availability—events that are easier to recall are judged to be more likely—was the major cause for increased cautiousness following recent droughts. Importantly, probabilistic predictions attenuated the impact of recency, inspired greater trust, and allowed participants to make better decisions overall than did deterministic predictions. Implications are discussed.
\end{abstract}

\section{Introduction}

To what degree are people's decisions about the future influenced by recent past events? For instance, are we more cautious after a recent negative experience? There are some prominent examples suggesting that this is the case. For instance, Hurricane Rita, occurring shortly after the historically destructive Hurricane Katrina, generated one of the largest hurricane evacuations in U.S. history. The response was twice what was anticipated by area evacuation plans and led to massive traffic jams and travel delays posing additional risks (Zhang et al. 2007). However, systematic studies of responses following major weather events are less conclusive. There is some evidence that purchasing flood insurance increases immediately following a flood event but declines after about 3 years if no additional floods are experienced (Atreya et al. 2015). However, survey studies of decisions to evacuate or seek shelter in the face of severe weather show mixed results. Among

Supplemental information related to this paper is available at the Journals Online website: https://doi.org/10.1175/WCAS-D19-0115.s1.

Corresponding author: Raoni Demnitz,rfgd@uw.edu respondents in affected areas, past experience can either increase, decrease, or have no influence on protective decisions (Huang et al. 2015; Lazo et al. 2015; Lindell 2012). This may be because of the great variability in past experience of respondents, including differences in outcome (perceived hit or false alarm), recency, intensity, and frequency of past experience with severe weather. Although some have argued that these problems can be at least partially overcome with better measurement techniques (Demuth 2018), here we take an experimental approach, holding these critical factors constant. Therefore, the first goal of the research reported here was to determine whether people make more cautious decisions following recent negative events in a controlled experimental setting.

Indeed, there is very little experimental research to support the impact of recent events on subsequent decisions. One exception is a line of research that compares learning about event likelihood from experience to learning from description. Although it does not speak to the recency question directly (nor was it designed to do so), it shows that people tend to underweight small probability events, for example, a $10 \%$ chance, when learning from experience as compared to description, because participants are less likely to have experienced rare events recently (Hertwig et al. 2004). In other 
words, this work suggests that people use event recency (or lack thereof in this case) to judge overall event likelihood.

A similar mechanism may underlie the hypothesized effect of overcautiousness following recent negative events. When they have experienced a negative event recently, people may assume that similar events are more likely to occur in the future. This is not a new idea and has been described as a form of attribute substitution (Kahneman and Frederick 2002). In such cases, people may substitute a computationally easier answer (recency) for a more accurate but computationally difficult answer (likelihood).

There are at least two broad cognitive mechanisms by which this might occur. The effects of recency could arise from an intuitive judgment in which the ease with which recent events are retrieved from memory (e.g., hurricane or flood) is automatically attributed to higher likelihood of such events, known as the availability heuristic (Kahneman et al. 1982). Alternatively, inferring likelihood from recency in natural settings might also stem from the conscious judgment that recent events are the beginning of a trend of increased likelihood for such events that will extend into the future. Therefore, the second goal of the research reported here was to shed light on the psychological mechanism to which the hypothesized effect of recency on decisionmaking is attributable.

The third goal of this research was to test whether providing explicit uncertainty information reduces any undue impact of recency on decision-making. If recent events distort people's perception of the likelihood of future events, it may cause them to make less optimal decisions. If so, then providing information about the actual likelihood of such events might reduce the impact of recency. Indeed, our own research suggests that people can understand and use explicit numeric uncertainty information to make better decisions than when deterministic forecasts alone are used (Joslyn and LeClerc 2012, 2013). In addition, probabilistic estimates appear to increase trust in the forecast and counteract the effect of recent false alarms on subsequent decisions. Participants were more willing to comply with warnings following false alarms when likelihood information was provided than when it was not (LeClerc and Joslyn 2015). In another experiment, participants thought floods were more likely and expressed greater concern when the same number of floods was experienced recently as compared to the more distant past. However, this effect disappeared when participants were given numeric flood probabilities (Grounds et al. 2018). Thus, there is reason to believe that providing explicit likelihood information may reduce a cognitive bias resulting from recency that impacts decision-making.

Therefore, the four experiments reported here tested the effect of event recency and explicit uncertainty information on participants' decisions in a laboratory-based seasonal climate task concerning drought. Experiment 1 sought to establish the effect of recency on decision-making by systematically manipulating when droughts occurred while holding constant drought frequency. Experiments 2-4 were designed to uncover the cognitive mechanisms by which the recency effect operated.

\section{Experiment 1}

\section{a. Method}

\section{1) PARticipants}

A total of 208 University of Washington psychology students $(51 \%$ female $)$ participated for course credit. The mean age was 19.26 years old $(\mathrm{SD}=1.62)$.

\section{2) Procedure}

Participants performed a computer-based crop choice task based on seasonal climate predictions. After providing informed consent and demographic information, they read instructions on the computer while the experimenter read the same instruction aloud. Each participant assumed the role of an international agricultural consultant hired by farmers to give advice on which crops to plant based on seasonal climate predictions. In this simplified task, there were two possible crops. The risky crop cost $\$ 100$ to plant and yielded $\$ 300$ in nondrought conditions and $\$ 0$ in drought. The riskless crop cost $\$ 200$ dollars but yielded $\$ 300$ regardless of whether drought was observed. In this simplified scenario, there were only two possible outcomes: drought or no drought.

Each participant received a virtual budget of $\$ 1,000$. Advice given to farmers had both costs and benefits that impacted the budget. An amount equivalent to the per acre cost of the crop advised was subtracted from the budget. An amount equivalent to the per-acre profit based on the outcome was added to the budget. The goal was to end the 46 trials with the highest possible budget. On each trial, representing a different hypothetical farmer advisee, participants received a prediction, decided which of the two crops to advise, and rated how much they trusted the prediction on a drop-down menu that included six options ranging from "not at all" to "completely." They next rated how concerned they were about the possibility of future drought on a dropdown menu that included six options ranging from "not 
TABLE 1. Drought predictions and outcomes by recency and prediction format in first half of trials.

\begin{tabular}{|c|c|c|c|c|c|c|}
\hline \multirow[b]{2}{*}{ Farmer client } & \multicolumn{3}{|c|}{ Recent drought condition } & \multicolumn{3}{|c|}{ Distant droughts condition } \\
\hline & Probabilistic & Deterministic & Observation & Probabilistic & Deterministic & Observation \\
\hline 1 & 30 & No & No & 30 & No & No \\
\hline 2 & 20 & No & No & 60 & Drought & Drought \\
\hline 3 & 20 & No & No & 50 & Drought & Drought \\
\hline 4 & 40 & Drought & No & 40 & Drought & Drought \\
\hline 5 & 20 & No & No & 30 & No & Drought \\
\hline 6 & 30 & No & No & 10 & No & Drought \\
\hline 7 & 40 & Drought & No & 50 & Drought & Drought \\
\hline 8 & 10 & No & No & 20 & No & Drought \\
\hline 9 & 10 & No & No & 10 & No & No \\
\hline 10 & 60 & Drought & No & 60 & Drought & No \\
\hline 11 & 10 & No & No & 10 & No & No \\
\hline 12 & 50 & Drought & No & 50 & Drought & No \\
\hline 13 & 30 & No & No & 30 & No & No \\
\hline 14 & 20 & No & No & 20 & No & No \\
\hline 15 & 10 & No & No & 10 & No & No \\
\hline 16 & 60 & Drought & Drought & 20 & No & No \\
\hline 17 & 50 & Drought & Drought & 20 & No & No \\
\hline 18 & 40 & Drought & Drought & 40 & Drought & No \\
\hline 19 & 30 & No & Drought & 20 & No & No \\
\hline 20 & 10 & No & Drought & 30 & No & No \\
\hline 21 & 50 & Drought & Drought & 40 & Drought & No \\
\hline 22 & 20 & No & Drought & 10 & No & No \\
\hline 23 & 30 & No & No & 30 & No & No \\
\hline
\end{tabular}

at all" to "extremely." Immediately after concern ratings, participants were informed of the outcome. The budget, shown on the screen at all times, was updated by subtracting crop costs and adding profits as soon as they occurred. Finally, participants were debriefed and thanked for their participation.

\section{3) STIMULi}

There was a total of seven droughts that occurred in sequence in the first half of trials. For half the participants, the drought sequence occurred in trials 16-22 (recent condition); for the other half, it occurred in trials 2-8 (distant condition). See Table 1 for trial characteristics. The second half of trials (24-46) were considered the target trials on which the impact of the manipulations was measured. In the second half of trials there were also seven droughts interspersed throughout in an identical order for all participants. Thus, the total number of droughts as well as the total droughts in the first half was identical for all participants, effectively holding drought frequency constant (droughts occurred on $30 \%$ of trials). In addition, there was an equal number of misses (no drought predicted but drought occurred), false alarms (drought predicted but did not occur), hits (drought predicted and occurred), and correct rejections (drought not predicted and did not occur) in the first and second halves of trials and for all participants.
Drought predictions were presented in two formats. Half the participants in each condition (recent or distant) were simply informed of whether or not drought was predicted for that region, for example, "In region 2, drought is predicted." This will be referred to as the deterministic prediction because it implies a single outcome. The other half of participants was informed of the percentage chance for drought, for example, "there is a $40 \%$ chance of drought" (probabilistic). Thus, participants were divided into four different groups (recent-deterministic, recent-probabilistic, distantdeterministic, distant-probabilistic).

Probabilistic predictions ranged from a $10 \%$ chance to a $60 \%$ chance of drought, in $10 \%$-chance increments. The percentage likelihoods for droughts were reliable, such that the observed frequency (e.g., $10 \%$ of trials resulted in drought) matched the prediction (e.g., 10\% chance). However, it is important to note that the underlying probability of drought was the same in both format conditions on any given trial. In other words, although they represent two different kinds of predictions, they were based on the same underlying information. ${ }^{1}$

\footnotetext{
${ }^{1}$ We focus on this probability range because it is of particular interest in the weather domain where, due to costly adverse outcomes, protective action is often required when the probability of the event is less than $50 \%$.
} 
TABLE 2. Expected profits associated with the risky crop and the riskless crop.

\begin{tabular}{ccc}
\hline \hline & \multicolumn{2}{c}{ Expected profit } \\
\cline { 2 - 3 }$P($ drought $)$ & Risky crop & Riskless crop \\
\hline $10 \%$ & $\$ 170$ & $\$ 100$ \\
$20 \%$ & $\$ 140$ & $\$ 100$ \\
$30 \%$ & $\$ 110$ & $\$ 100$ \\
$40 \%$ & $\$ 80$ & $\$ 100$ \\
$50 \%$ & $\$ 50$ & $\$ 100$ \\
$60 \%$ & $\$ 20$ & $\$ 100$ \\
\hline
\end{tabular}

The economically optimal strategy in this task was to choose the riskless crop whenever the percentage chance of drought was greater than $33 \%$ and to choose the risky crop otherwise (see Table 2). This is because, at 33\% chance of drought, the profit of the riskless crop (\$100) is greater than the expected value of the risky crop. The theoretical expected value of choosing the risky crop on any trial is, $(1-p$ [drought] $) \times \$ 300-\$ 100$. Therefore, if there were a $34 \%$ chance of drought, the expected profit would be, $(1-0.34) \times \$ 300)-\$ 100=\$ 98$. See Table A in the online supplemental material showing the performance (i.e., budget chance and balance) of a hypothetical participant making crop choices based on the aforementioned decision rule.

Although those in the deterministic condition did not see the probability of drought, the predictions they were given followed this decision rule. That is, drought was not predicted when the underlying percentage chance of drought was less than $33 \%$ (in practice less than $30 \%$ because only $10 \%$ increments were used) and drought was predicted when the probability was $40 \%$ or greater. Thus, although those in the deterministic condition received less specific information, it was, in a sense, the optimal information because it was economically advantageous to choose the riskless, drought resistant crop whenever drought was predicted. Notice that, as with many adverse weather events, cautiousness is required here at a relatively low chance of occurrence. That is because the potential negative consequences of the event far outweigh the cost of protection (this riskless crop in this case).

\section{4) Design}

A $2 \times 2$ complete factorial design was used with two between-group independent variables: recency (recent vs distant) and prediction format (deterministic vs probabilistic). Participants were randomly assigned to one of the four resulting conditions.

\section{5) HYPOTHESES}

We expected participants in the recent condition to be more concerned and more cautious in their subsequent crop choices than those in the distant condition, leading

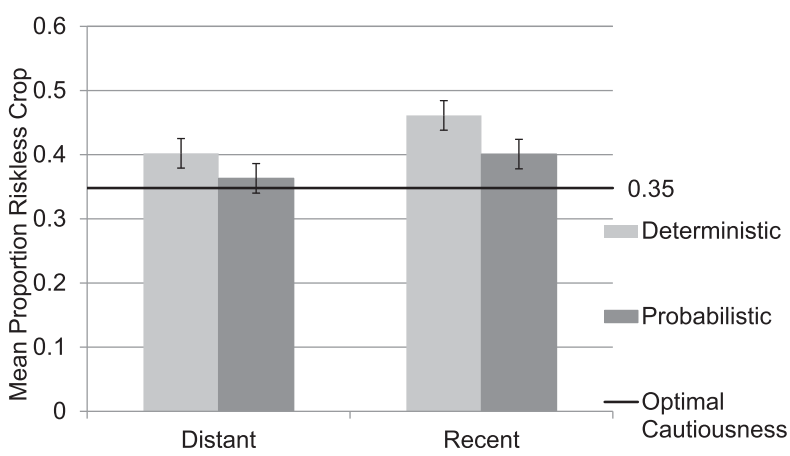

FIG. 1. Effects of recency and drought prediction format on riskless crop choice in experiment 1 . The optimal proportion of trials on which to choose the riskless crop is marked by the horizontal black line. The columns include standard error bars.

to suboptimal risk-averse decisions (choosing the riskless crop when the risky crop was economically advantageous), and yield a lower mean expected value. We expected participants receiving probabilistic predictions to express higher trust in the prediction, make decisions with higher expected value, and to be less influenced by recency than participants receiving deterministic forecasts.

\section{6) Results}

To determine the impact of recency and prediction format, we calculated four dependent variables: the mean percent of trials upon which the riskless crop was chosen (cautiousness), the mean expected value, the mean concern rating, and the mean trust rating. These were submitted to separate $2 \times 2$ between-group ANOVAs using and alpha level of 0.05 . Recency (recent vs distant) and prediction format (probabilistic vs deterministic) were the independent variables.

\section{(i) Cautiousness}

In support of our hypotheses, participants in the recent condition chose the riskless crop on a significantly larger proportion of trials (mean $M=0.43, \mathrm{SD}=0.15$ ) than participants in the distant condition $(M=0.38, \mathrm{SD}=$ $0.18), F(1,204)=4.38, p=0.038$ (Cohen's $D=0.29$ ). Participants with probabilistic predictions chose the riskless crop on a significantly smaller proportion of trials $(M=0.38, \mathrm{SD}=0.15)$ than participants with deterministic predictions $(M=0.43, \mathrm{SD}=0.18), F(1,204)=4.61, p=$ 0.03 (Cohen's $D=0.3$ ). The interaction between recency and prediction format did not reach significance. All groups chose the riskless crop more often than was optimal (0.35), suggesting an overall tendency to be risk averse (see Fig. 1).

\section{(ii) Expected value}

To determine whether decision quality was impacted, we examined expected value. Each decision was assigned 


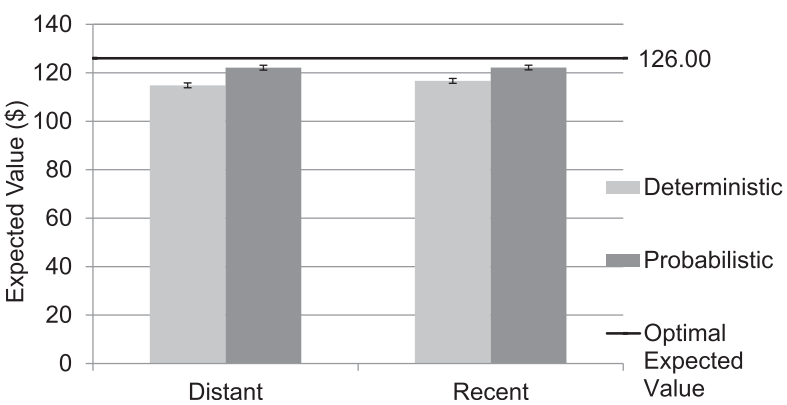

FIG. 2. Effects of recency and drought prediction format on expected value in experiment 1 . The optimal expected value $(\$ 126)$ is marked by the horizontal black line. The columns include standard error bars.

either the expected value of the risky crop it if was chosen or $\$ 100$ if the riskless crop was chosen. Then a mean was calculated for each participant. Participants with probabilistic predictions made decisions with significantly higher mean expected value $(M=\$ 122.15, \mathrm{SD}=\$ 5.29)$ than participants with deterministic predictions $(M=$ $\$ 115.72, \mathrm{SD}=\$ 8.66), F(1,204)=41.63, p<0.001$ (Cohen's $D=0.9$ ), suggesting that having explicit uncertainty information helped them to make better decisions (see Fig. 2). However, neither the effect of recency nor the interaction between recency and prediction format reached significance.

\section{(iii) Concern}

Participants with deterministic predictions had significantly higher mean concern ratings $(M=3.26, \mathrm{SD}=$ 1.07) than participants with probabilistic predictions $(M=2.98, \mathrm{SD}=0.71), F(1,204)=5.06, p=0.03$ (Cohen's $D=0.31$ ). Neither the effect of recency nor the interaction between recency and prediction format reached significance.

To better understand this unanticipated effect of prediction format on concern, we categorized the 46 trials into those in which drought was (probability $>$ $30 \%$ ) and was not predicted (probability $\leq 30 \%$ ) to which we refer as "drought prediction category." A mixed-model ANOVA with prediction format as the between-group variable and drought prediction category as the within-group variable revealed that participants were significantly more concerned when drought was predicted $(M=3.5, \mathrm{SD}=0.95)$, than when it was $\operatorname{not}(M=2.86, \mathrm{SD}=0.91), F(1,206)=95.31, p<0.001$ (Cohen's $D=1.36$ ). Importantly, prediction format interacted with drought-prediction category, $F(1,206)=$ $11.76, p=0.001$ (Cohen's $D=0.48$ ). When drought was predicted, participants had similar mean concern ratings regardless of prediction format (probabilistic: $M=3.54$, $\mathrm{SD}=0.76$; deterministic: $M=3.49, \mathrm{SD}=1.11$ ).

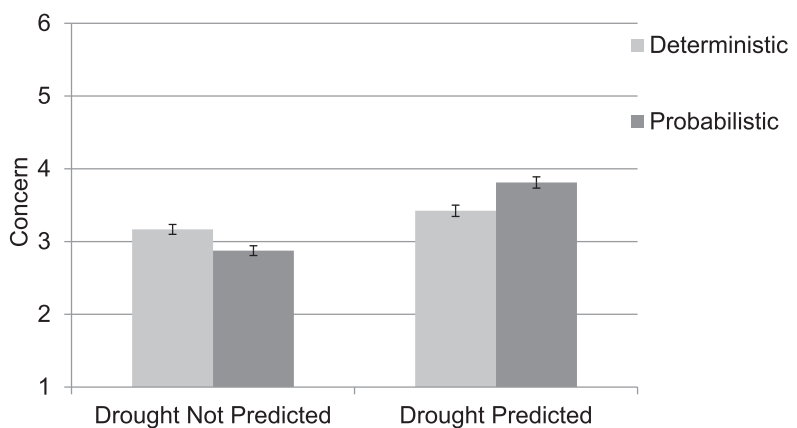

FIG. 3. Effects of drought prediction and drought prediction format on concern in experiment 1 . The columns include standard error bars.

However, when drought was not predicted, participants with probabilistic predictions expressed lower concern $(M=2.64$, SD $=0.74)$ than those given deterministic predictions $(M=3.08, \mathrm{SD}=1.02), t(206)=$ $3.59, p<0.001$ (Cohen's $D=0.5$; see Fig. 3).

\section{(iv) Trust}

Unexpectedly, recency effected trust such that participants in the recent condition trusted predictions significantly less $(M=3.21, \mathrm{SD}=1.0)$ than did participants in the distant condition $(M=3.53, \mathrm{SD}=1.0)$, $F(1,204)=5.51, p=0.02$ (Cohen's $D=0.33$ ). Although participants gave probabilistic predictions higher trust ratings $(M=3.49, \mathrm{SD}=1.0)$ than deterministic predictions $(M=3.24, \mathrm{SD}=1.0)$, this difference was only marginally significant, $F(1,204)=3.5, p=0.06$ (Cohen's $D=0.26$ ).

\section{b. Discussion for experiment 1}

In this laboratory-based experiment in which the overall rate of previous droughts was held constant, we found that the recency of drought caused users to make significantly more cautious choices in subsequent trials suggesting that they believed that future droughts were more likely. Granted there are many important differences between this laboratory-based experiment and real-world weather-related or seasonal climate decisions, including the magnitude of the consequence, the time frame and the surrounding circumstances. Nonetheless, it is important to note that in this experimental setting in which the critical extraneous variables were held constant, it is clear that recent events have a significant effect on subsequent decisions.

There are at least three psychological mechanisms that could account for this effect. It could simply be that droughts were more salient or available to participants' in the recent condition. If so, the process by which 
recency works may be largely unconscious. Ease of retrieval may automatically be taken as an indication of increased likelihood. Indeed, ease of retrieval is often due to increased frequency and can therefore be a valid indication of increased likelihood (Kahneman et al. 1982). However, in this case the number of previous droughts in the recent and distant conditions was equivalent. Therefore, if availability accounts for increased cautiousness in this paradigm it is due to the recency with which droughts were experienced alone.

An alternative, although not mutually exclusive, explanation for the increased cautiousness observed here is that participants in the recent condition made a conscious assessment that the region for which they were providing advice had experienced a shift in climate making it especially drought prone. For that reason, they opted to be more cautious.

A third possible explanation is that in the recent condition, increased cautiousness was the result of lack of trust in the prediction. Recall that there was lower trust in the recent as compared to the distant condition. Although overall accuracy (as well as the number of misses and false alarms) in the first half of trials was the same in recent and distant conditions, the predictions in the recent-drought condition resulted in three misses immediately preceding the second half of trials. Perhaps this reduced trust and led to overcautiousness. Experiment 2 was conducted to evaluate these three explanations.

Regardless of the cause of overcautiousness, it is important to note that the deleterious effect was attenuated by probabilistic predictions. Participants with probabilistic predictions were less overcautious, had a higher expected value, and reported better calibrated concern than did participants with deterministic predictions.

\section{Experiment 2}

To distinguish between the three possible explanations for overcautiousness in the recent condition of experiment 1 , we conducted a second experiment. Halfway through experiment 2, after trial 23, participants were informed that subsequent (target) trials would take place 5 years later and in a different geographic region from the previous trials. If the effect of recency was due to participants reasoning that their advisees were in a drought-prone region, the change in time and place should diminish the effect and decrease the difference in cautiousness between the distant and recent groups. Our prediction regarding the impact on trust was less clear. Change in location may also attenuate the impact on trust because participants in the recent condition may reason that the prediction for the new region might be more accurate. If, on the other hand, the effect of recency was due to availability in experiment 1 , we would expect the same increased cautiousness in the target trials in the recent condition in experiment 2 . That is because, regardless of the change in time and location, the recent drought events should be more salient in participants' memory than distant droughts.

A likelihood scale was added to experiment 2 to determine whether increased cautiousness was indeed due to an increased perception of the likelihood of drought rather than lack of trust in the prediction. If the mechanism behind the increase in cautiousness was distrust in the prediction, then likelihood ratings should not differ systematically between the recent and distant conditions. However, both other explanations-increased availability or reasoning that the region was drought prone-should be accompanied by a perception of increased likelihood of droughts in the future.

\section{a. Method}

\section{1) PARticipants}

A total of 640 University of Washington psychology students $(54.3 \%$ female) participated for course credit. The mean age was 19 years old $(\mathrm{SD}=1.58)$ and ranged between 17 and 39 years old. ${ }^{2}$

\section{2) Procedure}

The procedure was identical to experiment 1 except that the 46 trials were presented in two halves. After trial 23, participants were told that 5 years had passed, the firm had expanded to a different continent and the participant had been assigned a new set of farmer-clients there. To emphasize this change, the virtual budget was divided so that half $(\$ 500)$ was provided at the beginning of the experiment and the other half $(\$ 500)$ at the break. Budget amounts remaining at the end of the first 23 trials did not carry over to the second set of trials so that the two halves were distinct. On each trial, following the presentation of the drought prediction, participants rated the likelihood of drought on an unmarked slider with the anchors "impossible" and "certain." The stimuli and design were identical to those in experiment 1.

\section{3) RESUlts}

The data summary procedures and analyses for experiment 2 were identical to those used in experiment 1 .

\footnotetext{
${ }^{2}$ In this experiment and the next, we also tested the effects of visual stimuli in a between-group manipulation, hence the larger sample size. However, no effects were detected, and in the interest of brevity we omitted this manipulation.
} 


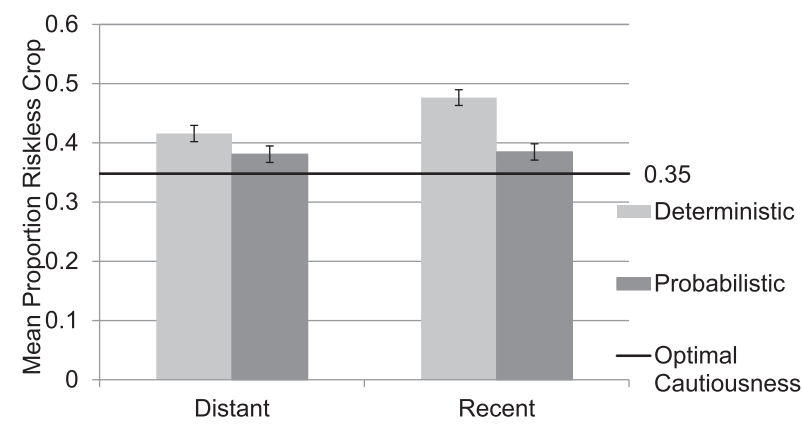

FIG. 4. Effects of recency and drought prediction format on riskless crop choice in experiment 2. The optimal proportion of trials on which to choose the riskless crop is marked by the horizontal black line. The columns include standard error bars.

\section{(i) Cautiousness}

As with experiment 1 , despite the break screen describing a change in location, participants in the recent condition chose the riskless crop on a significantly higher proportion of trials $(M=0.43, \mathrm{SD}=0.18)$ than participants in the distant condition $(M=0.40, \mathrm{SD}=0.17)$, $F(1,636)=5.58, p=0.02$ (Cohen's $D=0.19$ ), although the effect size was smaller than that observed in experiment 1 (Cohen's $D=0.29$ ). As in experiment 1 participants with probabilistic predictions chose the riskless crop on a lower proportion of trials $(M=0.38, \mathrm{SD}=0.15)$ than participants with deterministic predictions $(M=0.45, \mathrm{SD}=0.19)$, $F(1,636)=21.52, p<0.001$ (Cohen's $D=0.37$ ). In addition, there was a significant interaction between recency and prediction format, $F(1,636)=4.31, p=0.04$ (Cohen's $D=0.17)$. In the recent condition, participants with probabilistic predictions chose the riskless crop on a significantly lower proportion of trials $(M=0.39, \mathrm{SD}=0.16)$ than participants with deterministic predictions $(M=0.48$, $\mathrm{SD}=0.19), t(325)=4.84, p<0.001$ (Cohen's $D=0.57$, although there was no difference between prediction formats in the distant condition (see Fig. 4).

\section{(ii) Expected value}

In experiment 2 the effect of recency on expected value reached significance. Mean expected value was significantly lower in the recent $(M=\$ 117.81, \mathrm{SD}=\$ 8.15)$ than in the distant condition $(M=\$ 119.06, \mathrm{SD}=\$ 7.57)$, $F(1,636)=4.12, p=0.04$ (Cohen's $D=0.16$ ). As with experiment 1 , mean expected value was significantly higher in the probabilistic $(M=\$ 121.10, \mathrm{SD}=\$ 6.33)$ than in the deterministic condition $(M=\$ 115.85, \mathrm{SD}=\$ 8.38)$, $F(1,636)=79.29, p<0.001$ (Cohen's $D=0.71$; see Fig. 5).

\section{(iii) Concern}

There was a small but significant effect of recency on concern. Surprisingly, it was in the opposite direction to

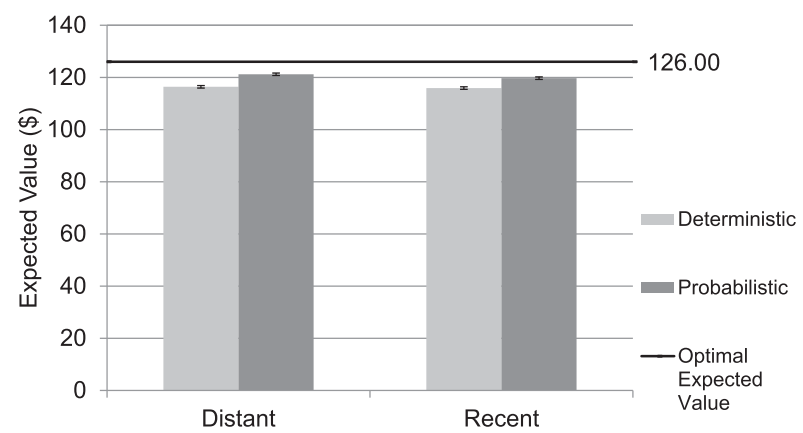

FIG. 5. Effects of recency and drought prediction format on expected value in experiment 2 . The optimal expected value $(\$ 126)$ is marked by the horizontal black line. The columns include standard error bars.

what one would expect. Participants in the distant condition were more concerned about droughts $(M=3.27, \mathrm{SD}=0.71)$ than participants in the recent condition $(M=3.16, \mathrm{SD}=0.67), F(1,636)=4.33$, $p=0.04$ (Cohen's $D=0.17$ ). However, as with experiment 1 , participants in the probabilistic condition were significantly less concerned $(M=3.12$, $\mathrm{SD}=0.61)$ than those in the deterministic condition $(M=3.31, \mathrm{SD}=0.75) F(1,636)=11.83, p<0.001$ (Cohen's $D=0.27$ ).

We categorized the 46 trials into those in which drought was predicted (i.e., greater than $30 \%$ chance), and was not predicted (i.e., $30 \%$ chance or less), and conducted a mixed-model ANOVA with prediction format as the between-subject variable and drought prediction category as the within-subject variable. The results were similar to those observed in experiment 1 . Participants were significantly more concerned when drought was predicted $(M=3.62, \mathrm{SD}=0.78)$ than when drought was not predicted $(M=3.01, \mathrm{SD}=0.75)$, $F(1,638)=380.21, p<0.001$ (Cohen's $D=1.54$ ), and there was a significant interaction between prediction format and drought prediction category, $F(1,638)=$ $80.88, p<0.001$ (Cohen's $D=0.71$ ). When drought was predicted, participants with probabilistic predictions were more concerned than participants with deterministic predictions $(M=3.54, \mathrm{SD}=0.86), t(638)=2.79$, $p<0.001$ (Cohen's $\mathrm{D}=0.21$ ). However, when drought was not predicted, participants with probabilistic predictions $(M=2.81, \mathrm{SD}=0.68)$ were less concerned than those with deterministic predictions $(M=3.20, \mathrm{SD}=$ $0.77), t(638)=6.83, p<0.001$ (Cohen's $D=0.55$ ), suggesting better calibration of concern among those with probabilities (see Fig. 6).

\section{(iv) Trust}

As with experiment 1 probabilistic predictions inspired significantly higher trust $(M=3.42, \mathrm{SD}=0.80)$ 


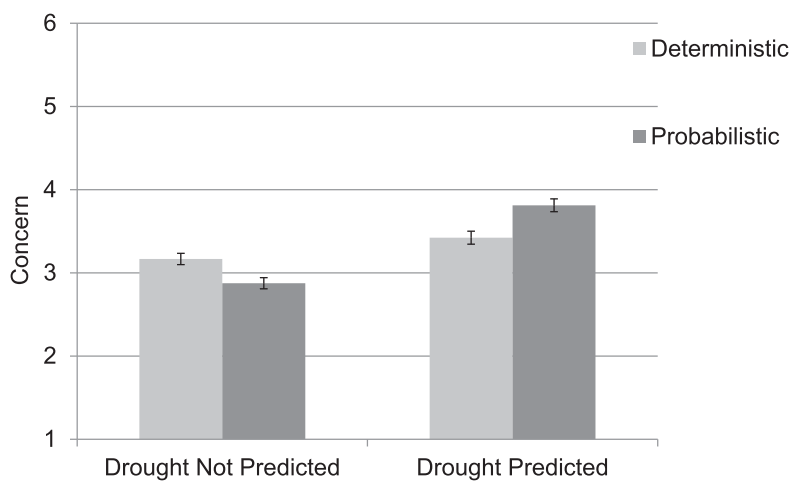

FIG. 6. Effects of drought prediction and drought prediction format on concern in experiment 2 . The columns include standard error bars.

than deterministic predictions $(M=3.18, \mathrm{SD}=0.85)$, $F(1,636)=14.08, p<0.001$ (Cohen's $D=0.3$ ). However, the effect of recency on trust was not significant, contradicting the theory entertained in experiment 1 that lack of trust in the prediction was responsible for the increased cautiousness in the recent condition. The interaction between prediction format and recency did not reach significance.

\section{(v) Likelihood}

Contrary to our hypothesis, participants in the recent condition did not rate droughts as significantly more likely than those in the distant condition, although they made more cautious decisions. However, participants with deterministic predictions rated drought as significantly more likely $(M=43.86 \%, \mathrm{SD}=13.11 \%)$ than those with probabilistic predictions $(M=33.74 \%, \mathrm{SD}=$ $8.17 \%), F(1,636)=135.12, p<0.001$ (Cohen's $D=$ 0.92 ), perhaps explaining the increase in concern and cautiousness among those using deterministic predictions. It is also important to note that the mean likelihood rating of those with probabilistic forecasts was closer to the actual mean likelihood of drought $(30 \%)$, suggesting that they had a better understanding of the likelihood of drought.

\section{b. Discussion for experiment 2}

In experiment 2 as with experiment 1 , those with probabilistic predictions made better decisions, in terms of expected value, than did those using deterministic predictions. In addition, they had higher trust in the prediction. This may have been because probabilistic predictions provided them with a better understanding of the likelihood of drought, revealed in participants' likelihood ratings, which were closer to the actual percentage chance of drought in the simulation. Moreover, as with experiment 1 , probabilistic predictions helped to better calibrate participants' concern levels compared to deterministic predictions.

Importantly, in experiment 2 the increase in cautiousness in the recent condition persisted. This occurred despite the change in time and location introduced prior to the target trials, suggesting that the effect was not due to reasoning that advisees were in a drought-prone region. However, there was no significant difference in trust between the recent and distant groups, suggesting that the overcautiousness is not attributable to lack of trust in the forecast.

In addition, increased cautiousness translated into a decrease in expected value, demonstrating that the effect has a negative impact on decision-making overall. Once again, the predominant error was a risk-averse error as has been seen in other work in which gains are involved (Kahneman and Tversky 1979). Here, participants tended to be too cautious and advised the low-yield riskless crop too often. This error increased when droughts were recent, decreasing expected overall value. It is important to note, however, that the effect size of overcautiousness observed in experiment 2 was smaller than that observed in experiment 1 . This may have been because there were two mechanisms operating in experiment 1 , a causal-reasoning process by which participants deduced a climatic trend toward drought (disrupted in experiment 2 by the "change in venue" message), as well as an intuitive availability heuristic that persisted in experiment 2 despite the midexperiment message. In other words, the larger effect of recency in experiment 1 may have been a combination of two processes.

Alternatively, the larger effect of recency in experiment 1 could be due entirely to an availability mechanism that dissipated over the time it took participants to read the material on the break screen in experiment 2 . In support of this interpretation, and contrary to our hypothesis, recency did not influence likelihood ratings, suggesting that participants in the recent condition did not consciously believe that droughts were more likely than did participants in the distant condition. In addition, participants in the recent condition expressed less rather than more concern. This issue will be discussed in the conclusion. Taken together, these results suggest that the recency effect observed here was due at least in part to an intuitive mechanism such as availability.

\section{Experiment 3}

Experiment 3 was conducted to distinguish between two possible explanations for the reduction in the effect size of overcautiousness in the recent condition observed in experiment 2 as compared to the effect size in 
experiment 1: 1) A combination of two mechanisms were operating in experiment 1 , a causal-reasoning process as well as an intuitive availability heuristic but only the latter in experiment 2.2) an availability mechanism that was strong in experiment 1 when the target trials followed immediately after the manipulation but dissipated due to the break screen in experiment 2 reducing the effect size. To distinguish between these two hypotheses, experiment 3 was conducted. It was identical to experiment 2 with the exception that participants were informed, in the message delivered halfway through the experiment after trial 23 , that they were in the same general region and in the same calendar year. If participants in the recent condition were influenced by reasoning that it is a drought-prone region, then they should continue to be more cautious and the effect size should be comparable here to that of experiment 1 , because the same circumstances prevail before and after the message. However, if participants had been influenced by availability alone and it dissipated quickly over the time the midexperiment message was presented in experiment 2 , we expected to see a comparable small effect size in experiment 3.

\section{a. Method}

\section{1) PARTICIPANTS}

A total of 880 University of Washington psychology students $(62 \%$ female) participated for course credit. The mean age was 19 years old $(\mathrm{SD}=1.62)$ and ranged between 17 and 50 years old.

\section{2) Procedure}

The procedure was identical to that of experiment 2 except that the message presented after trial 23 , and prior to the target trials, informed participants that they were working in the same region and calendar year. The stimuli and design were identical to the previous two experiments.

\section{3) Results}

The data summary procedures and analyses for experiment 3 were identical to those used in experiment 2 .

\section{(i) Cautiousness}

Unlike the previous two experiments, participants had a similar proportion of riskless crop choices in the recent $(M=0.41, \mathrm{SD}=0.15)$ and distant conditions $(M=0.41, \mathrm{SD}=0.17), F(1,876)=0.06, p=0.81$ (Cohen's $D=0.00$ ). As in the previous two experiments, participants with probabilistic predictions chose the riskless crop on a lower proportion of trials $(M=0.39, \mathrm{SD}=$ $0.16)$ than participants with deterministic predictions

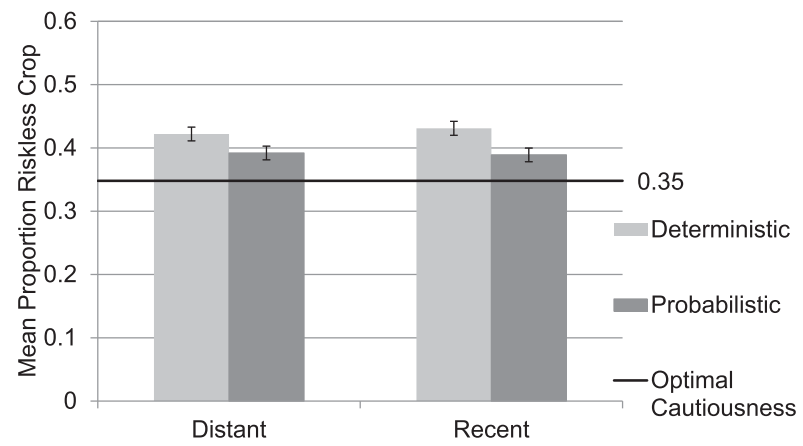

FIG. 7. Effects of recency and drought prediction format on riskless crop choice in experiment 3 . The optimal proportion of trials on which to choose the riskless crop is marked by the horizontal black line. The columns include standard error bars.

$(M=0.43, \mathrm{SD}=0.16), F(1,876)=10.99, p=0.001$ (Cohen's $D=0.22$; see Fig. 7). The interaction was not significant.

\section{(ii) Expected value}

As with experiment 2, participants in the recent condition had a significantly lower expected value $(M=$ $\$ 117.54, \mathrm{SD}=9.16)$ than those in the distant condition $(M=\$ 118.81, \mathrm{SD}=7.53), F(1,876)=5.59, p=0.018$ (Cohen's $D=0.16$ ). Again, participants with probabilistic predictions had a significantly higher expected value $(M=\$ 120.46 \mathrm{SD}=6.6)$ than participants with deterministic predictions $(M=\$ 115.88, \mathrm{SD}=9.34)$, $F(1,876)=70.96, p<0.001$ (Cohen's $D=0.57$; see Fig. 8 ).

\section{(iii) Concern}

As with the previous two experiments, participants with deterministic predictions were more concerned about droughts $(M=3.29, \mathrm{SD}=0.82)$ than participants with probabilistic predictions $(M=3.08, \mathrm{SD}=0.67)$, $F(1,876)=17.93, p<0.001$ (Cohen's $D=0.29$ ). There was no significant effect of recency or interaction.

Again, we categorized the 46 trials into those in which drought was predicted (i.e., greater than $30 \%$ chance of drought), and was not predicted (i.e., $30 \%$ chance or less of drought), and conducted a mixed-model ANOVA on concern with prediction format as the between-subject variable and drought prediction category as the withinsubject variable. The results were similar to the previous two experiments. Participants were significantly more concerned when drought was predicted $(M=3.58$, $\mathrm{SD}=0.81)$ than when it was not $(M=2.93, \mathrm{SD}=0.78)$, $F(1,878)=562.40, p<0.001$ (Cohen's $D=1.6$ ), and there was a significant interaction between prediction format and drought prediction category, $F(1,878)=$ 65.11, $p<0.001$ (Cohen's $D=0.55$ ). When drought was predicted participants were equally concerned but 


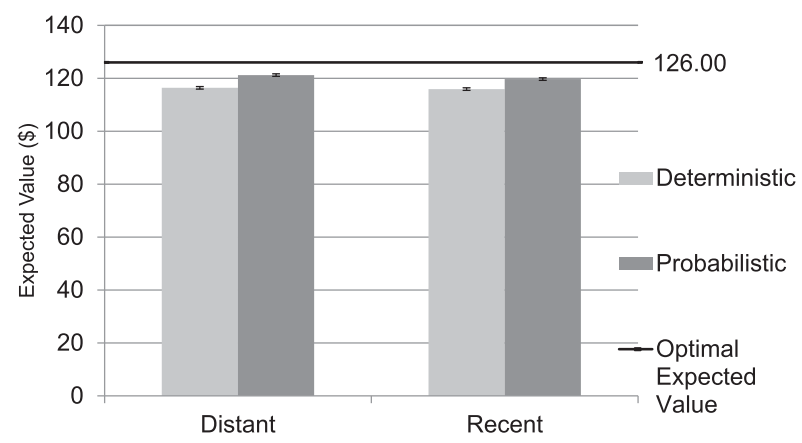

FIG. 8. Effects of recency and drought prediction format on expected value in experiment 3. The optimal expected value $(\$ 126)$ is marked by the horizontal black line. The columns include standard error bars.

when drought was not predicted participants with deterministic predictions were more concerned $(M=3.13$, $\mathrm{SD}=0.8)$ than participants with probabilistic predictions $(M=2.73, \mathrm{SD}=0.8), t(878)=7.84, p<0.001$ (Cohen's $D=0.53$; see Fig. 9).

(iv) Trust

As with the previous two experiments, probabilistic predictions inspired higher trust $(M=3.33, \mathrm{SD}=0.87)$ than deterministic predictions $(M=3.14, \mathrm{SD}=0.86)$, $F(1,876)=11.14, p=0.001$ (Cohen's $D=0.230$ ). As with experiment 1 (but not experiment 2) participants in the recent condition gave predictions lower trust ratings $(M=3.16, \mathrm{SD}=0.82)$ than participants in the distant condition $(M=3.31, \mathrm{SD}=0.91), F(1,876)=7.03, p=$ 0.01 (Cohen's $D=0.18$ ).

\section{(v) Likelihood}

As with experiment 2, there was no significant impact of recency on likelihood ratings. Mean drought likelihood ratings among participants in the recent condition $(M=37.82 \%, \mathrm{SD}=11.46)$ were similar to those in the distant droughts condition $(M=37.69 \%$, SD $=11.94)$, $F(1,876)=0.048, p=0.83$ (Cohen's $D=0)$. As with experiment 2 , the mean drought likelihood rating in the probabilistic condition $(M=33.89 \%$, SD $=9.37)$ was lower, and closer to the actual mean drought likelihood (30\%), than in the deterministic condition $(M=$ $41.66 \%, \mathrm{SD}=12.51), F(1,876)=108.59, p<0.001$ (Cohen's $D=0.73$ ).

\section{b. Discussion for experiment 3}

In experiment 3 , in which the break screen prior to target trials specified the same time and region, the overcautiousness effect in the recent condition disappeared. This suggests that the effect observed in the previous experiments was not due entirely to reasoning

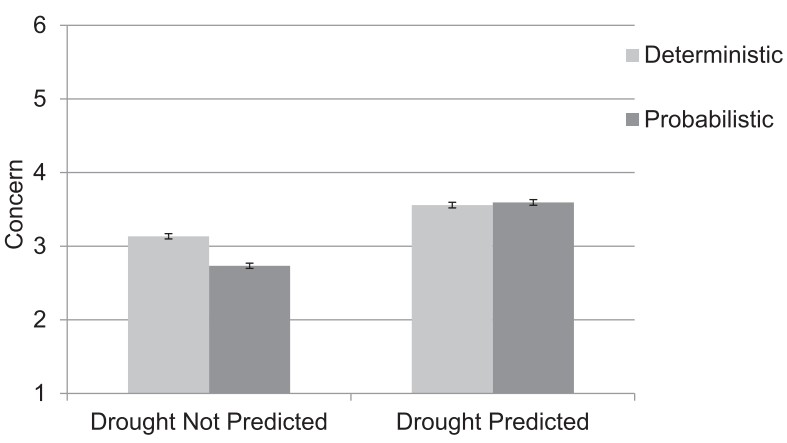

FIG. 9. Effects of drought prediction and drought prediction format on concern in experiment 3 . The columns include standard error bars.

that advisees were in drought-prone regions. If that had been the case, we would expect to see greater overcautiousness in experiment 3 compared to experiment 2 in which a change in time and region was introduced.

Therefore, taken together these results suggest that the effect of recency on cautiousness, when it is observed, might be due to an availability effect that is both intuitive and fragile in that it dissipates quickly over time. The time it took to show the midexperiment message, regardless of its content, may have been enough to reduce its effect compared to experiment 1 in which there was no break. Before drawing firm conclusions however, we thought it was important to replicate the effects of experiment 1.

\section{Experiment 4}

Experiment 4 was conducted to attempt to replicate the significant effect of recency on cautiousness observed in experiment 1 by removing the midexperiment break screen instruction. We also removed the likelihood rating that had been added in experiment 2 .

\section{a. Method}

\section{1) PARTICIPANTS}

A total of 298 University of Washington psychology students (57.2\% female) participated for course credit. The mean age was 19.08 years old $(\mathrm{SD}=2.01)$ and ranged between 18 and 45 years old.

\section{2) Procedure}

The procedure, stimuli, and design were identical to experiment 1 .

\section{3) Results}

The data summary procedures and analyses for experiment 4 were identical to those used in previous experiments. 


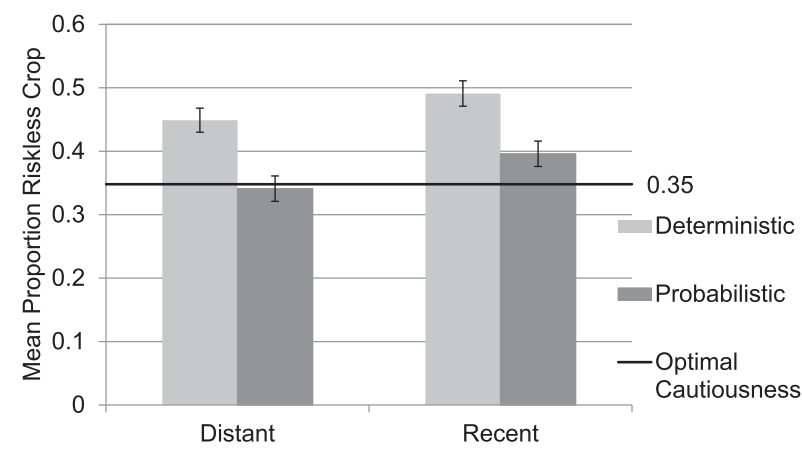

FIG. 10. Effects of recency and drought prediction format on riskless crop choice in experiment 4 . The optimal proportion of trials on which to choose the riskless crop is marked by the horizontal black line. The columns include standard error bars.

\section{(i) Cautiousness}

In support of our hypothesis and the results of experiments 1 and 2, participants in the recent condition chose the riskless crop on a significantly higher proportion of trials $(M=0.44, \mathrm{SD}=0.15)$ than participants in the distant condition $(M=0.40, \mathrm{SD}=0.19), F(1,288)=$ $6.09, p=0.01$ (Cohen's $D=0.29$ ). Again, participants with probabilistic predictions chose the riskless crop on a smaller proportion of trials $(M=0.37, \mathrm{SD}=0.13)$ than participants with deterministic predictions $(M=$ $0.47, \mathrm{SD}=0.20), F(1,288)=27.03, p<0.001$ (Cohen's $D=0.61$; see Fig. 10).

\section{(ii) Expected value}

As in experiment 1 , the effect of recency on expected value did not reach significance. However, the effect of prediction format on expected value, now seen in all four experiments, remained strong. Participants with probabilistic predictions had significantly higher expected value $(M=\$ 121.91, \mathrm{SD}=\$ 4.69)$ than did participants with deterministic predictions $(M=\$ 114.27$, $\mathrm{SD}=\$ 9.04) F(1,288)=81.66, p<0.001$ (Cohen's $D=1.07$; see Fig. 11).

\section{(iii) Concern}

As in experiment 1 , the effect of recency on concern was not significant. However, as with all three previous experiments, participants with deterministic predictions were significantly more concerned $(M=3.39, \mathrm{SD}=$ $0.91)$ than participants with probabilistic predictions $(M=3.11, \mathrm{SD}=0.81), F(1,288)=7.46, p=0.01$ (Cohen's $D=0.32$ ).

Again, trials were categorized into those in which drought was predicted (i.e., greater than $30 \%$ chance of drought) and was not predicted (i.e., $30 \%$ chance or less of drought). A mixed-model ANOVA on concern with prediction format as the between-subject variable and

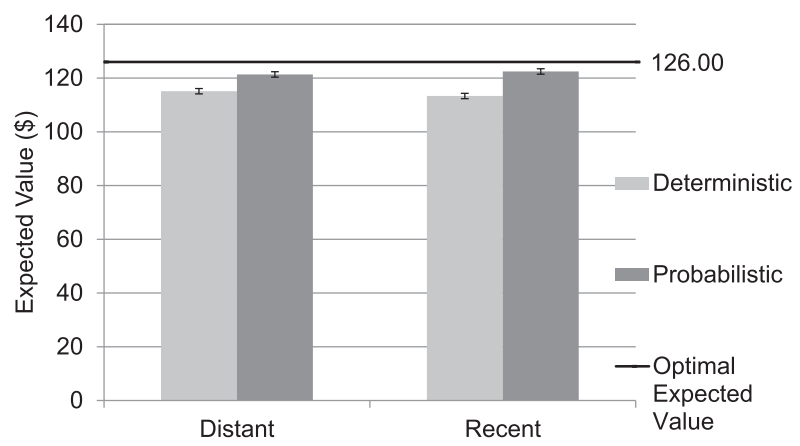

FIG. 11. Effects of recency and drought prediction format on expected value in experiment 4. The optimal expected value (\$126) is marked by the horizontal black line. The columns include standard error bars.

drought prediction category as the within-subject variable revealed that participants were significantly more concerned when drought was predicted $(M=3.59$, $\mathrm{SD}=0.93)$ than when it was not predicted $(M=3.02$, $\mathrm{SD}=0.91), F(1,290)=130.17, p<0.001$ (Cohen's $D=1.34)$. The interaction between prediction format and drought prediction category was significant, $F(1$, $290)=39.56, p<0.001$ (Cohen's $D=0.74$ ). While there was no significant difference in concern as a function of prediction format when drought was predicted (probabilistic: $M=3.64$, SD $=0.90$; deterministic: $M=3.53, \mathrm{SD}=1.0$ ), when drought was not predicted participants in the deterministic condition were significantly more concerned $(M=3.27$, $\mathrm{SD}=$ $0.88)$ than participants in the probabilistic condition $(M=2.74, \mathrm{SD}=0.86), t(290)=5.20, p<0.001$ (Cohen's $D=0.60$; see Fig. 12).

\section{(iv) Trust}

Unlike experiment 1 there was no significant effect of recency on trust. However, as in all previous experiments, trust was significantly higher in the probabilistic $(M=3.42, \mathrm{SD}=0.88)$ than in the deterministic $(M=$ $3.15, \mathrm{SD}=0.90)$ condition, $F(1,288)=6.55, p=0.01$ (Cohen's $D=0.3$ ).

\section{b. Discussion for experiment 4}

In experiment 4 , in which both the break screen and likelihood ratings were removed, the overcautiousness effect in the recent droughts condition reappeared. Moreover, the effect size was comparable to that observed in experiment 1 . We hypothesized that if the overcautiousness effect in the preceding three experiments was due to an intuitive availability process that is fragile and prone to interference, then it would reappear in the current experiment in which the break between droughts and target trials was removed. Indeed, 


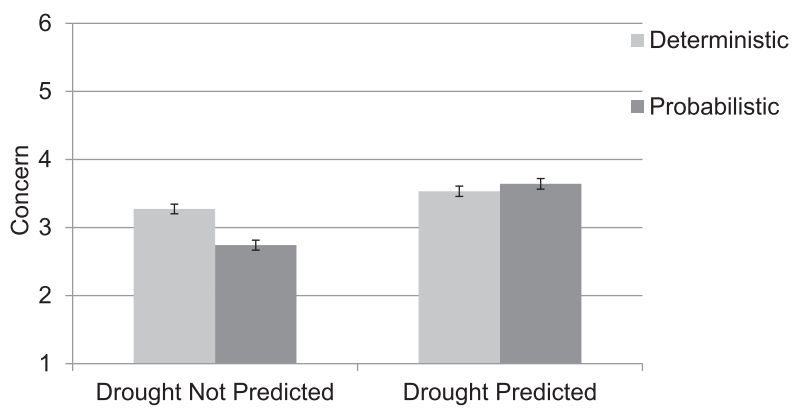

FIG. 12. Effects of drought prediction and drought prediction format on concern in experiment 4 . The columns include standard error bars.

in experiment 4 , when all procedures matched experiment 1 exactly, the increased cautiousness in the recent condition was replicated. We continue discussion of the implications of this result in the conclusion. Importantly, once more we found that probabilistic predictions were more trustworthy, allowed participants to better calibrate concern, reduced cautiousness, and increased expected value when compared to deterministic predictions.

\section{Conclusions}

The research reported here had three goals. The first was to determine whether people make more cautious decisions following recent negative events in a controlled experimental setting. Indeed, significant overcautiousness was observed after recently experiencing a series of droughts, compared to when the same number of droughts was experienced earlier, in experiments 1 , 2 , and 4 .

Our second goal was to shed light on the psychological mechanisms that might give rise to the effect of recent negative events on decision-making. We entertained three hypotheses about the cognitive mechanisms that underlie the recency effect observed here: (i) droughts were more available to memory, making future droughts seem more likely; (ii) reasoning that the region was drought prone; (iii) distrusting the predictions due to experiencing more recent false alarms. The recency effect was reduced when the break screen in experiment 2 introduced a temporal and geographic change. This suggested, initially, that the previously observed effect was due at least in part to participants in the recent condition thinking that the region was drought prone, reasoning that was contradicted by the information on the break screen. However, the recency effect was also reduced in experiment 3 in which the break screen described no change, contradicting the "reasoning" explanation. The fact that there was no increase in likelihood or concern ratings in the recent condition when overcautiousness was observed also tends to argue against a deliberate reasoning process, which would be expected to affect these variables as well. Interestingly, in the one experiment detecting a significant effect of recency on concern, the effect was in the opposite direction to that expected, with higher concern ratings in the distant rather than the recent condition, perhaps suggesting a "drought is due" effect (see Grounds et al. 2018). In addition, we ruled out the trust hypothesis because the reduction in trust in the recent condition did not reach significance in two of the three experiments in which overcautiousness was observed, including the replication experiment.

Therefore, the most plausible explanation for the impact of recency on cautiousness observed here is an intuitive, automatic process that made droughts slightly more available to memory immediately after they were experienced and was disrupted by the information on the break screens in experiments 2 and 3 reducing the effect in those experiments.

Our third goal was to test whether providing explicit uncertainty information reduces any undue impact of recency on decision-making. All four experiments indicate a strong and consistent advantage for predictions that include numeric probabilities. Although one might suspect that these benefits are confined to those with a university education, recent evidence suggests that this is not true (Grounds et al. 2017). Indeed, comparable benefits are seen among those with a high school education or less (Grounds and Joslyn 2018). Moreover, similar benefits are seen for climate predictions among a wide range of political ideologies (Joslyn and Demnitz 2019; Joslyn and LeClerc 2016). In the experiments reported here, not only did probabilistic predictions increase trust in the prediction, but they also allowed participants to better calibrate concern, reduced overcautiousness, and increased expected value compared to deterministic predictions. This suggests numeric uncertainty estimates such as this can be used not only to promote better decisions and greater trust but also to overcome common biases such as the availability heuristic. A summary of the overall results from these experiments can be found in Tables B and C in the supplemental materials.

Overcautiousness in the deterministic condition revealed that participants were not taking the deterministic prediction at face value. They clearly thought, despite the "no drought" prediction, that there was some chance of drought, which led them to select the riskless crop more often than was optimal and more often than those in the probabilistic condition. Recall that drought 
prediction in the deterministic condition was based on the optimal strategy such that, had participants followed the implicit advice, choosing the riskless crop only when drought was predicted, they would have achieved optimal expected value. Obviously, they did not. This result is particularly relevant because the deterministic drought prediction tested here is similar to the warnings people receive for a wide range of real-world events, which are designed to minimize misses by warning at lower likelihoods. Because experts are often reluctant to trust members of the public with probabilistic information, they provide binary advice or warnings when the estimated risk threshold of the average user has been reached. However, here, unlike the real-world setting, the probability threshold for predicting drought was perfectly matched to the users' optimal threshold as specified by the goals and economic structure of the task. Nonetheless, participants in the deterministic condition did not tend to follow the implied strategy, perhaps because they did not trust the prediction. Indeed, trust ratings were lower in the deterministic as compared to probabilistic conditions in all four experiments. This is in line with evidence that people are generally aware of the underlying uncertainty accompanying ostensibly deterministic forecasts (Joslyn and Savelli 2010; Morss et al. 2008). When uncertainty is omitted, people may simply find the prediction less plausible.

Taken together, the results of these experiments suggest that people can be overly influenced by recent events, leading them to make suboptimal decisions in some situations. We address some potential limitations next.

One might argue that the effects of recency on cautiousness may be overcome to some degree by expertise (e.g., actual agricultural experts). Here, remember that participants were university students, comparable to everyday users, rather than experts. However, there is also evidence that experts are susceptible to many of the same biases as are laypeople (Kahneman and Klein 2009; Tetlock 2017; Tetlock and Gardner 2015). Nonetheless, this work makes it clear that providing explicit uncertainty information can overcome the recency bias, even among the nonexperts tested here.

Although there are many important differences between this laboratory-based experiment and real-world weather-related or seasonal climate decisions, this experimental setting in which the critical extraneous variables were held constant allowed us to establish the effects of recency on protective decisions. Moreover, this work also indicates that availability likely plays a major role in the overcautiousness observed here, suggesting that people may not be fully aware of the impact of recency on their decisions. Thus, people may protect themselves unnecessarily as was shown here, leading to a reduction in trust for future warnings. On the other hand, they may fail to protect themselves in other situations in which dangerous events are predicted but have not been recently experienced. This is particularly critical in light of the increase of unprecedented extreme weather events expected to accompany climate change.

This work has important implications for a wide range of risky decision contexts such as finance, health, and personal safely (e.g., identity theft), in which risk perception may be unduly influenced by recent events. Importantly, the results reported here suggest an effective risk communication strategy for counteracting such effects. The evidence presented here suggests that numeric uncertainty information gives people a more accurate understanding of the likelihood of critical events both when they might be influenced by availability and, more generally, than do conventional deterministic formats, and helps them to make better decisions.

Acknowledgments. Funding for the research described here was provided by the National Science Foundation (NSF) Award SES-1430781 AM002.

\section{REFERENCES}

Atreya, A., S. Ferreira, and E. Michel-Kerjan, 2015: What drives households to buy flood insurance? New evidence from Georgia. Ecol. Econ., 117, 153-161, https://doi.org/10.1016/ j.ecolecon.2015.06.024.

Demuth, J. L., 2018: Explicating experience: Development of a valid scale of past hazard experience for tornadoes. Risk Anal., 38, 1921-1943, https://doi.org/10.1111/risa.12983.

Grounds, M. A., and S. L. Joslyn, 2018: Communicating weather forecast uncertainty: Do individual differences matter? J. Exp. Psychol. Appl., 24, 18-33, https://doi.org/10.1037/xap0000165.

- S. Joslyn, and K. Otsuka, 2017: Probabilistic interval forecasts: An individual differences approach to understanding forecast communication. Adv. Meteor., 2017, 3932565, https:// doi.org/10.1155/2017/3932565.

_, J. E. LeClerc, and S. Joslyn, 2018: Expressing flood likelihood: Return period versus probability. Wea. Climate Soc., 10, 5-17, https://doi.org/10.1175/WCAS-D-16-0107.1.

Hertwig, R., G. Barron, E. U. Weber, and I. Erev, 2004: Decisions from experience and the effect of rare events in risky choice. Psychol. Sci., 15, 534-539, https://doi.org/ 10.1111/j.0956-7976.2004.00715.x.

Huang, S. K., M. K. Lindell, and C. S. Prater, 2015: Who leaves and who stays? A review and statistical meta-analysis of hurricane evacuation studies. Environ. Behav., 48, 991-1029, https:// doi.org/10.1177/0013916515578485.

Joslyn, S., and S. Savelli, 2010: Communicating forecast uncertainty: Public perception of weather forecast uncertainty. Meteor. Appl., 17, 180-195, https://doi.org/10.1002/ met.190.

, and J. LeClerc, 2012: Uncertainty forecasts improve weatherrelated decisions and attenuate the effects of forecast error. J. Exp. Psychol. Appl., 18, 126-140, https://doi.org/10.1037/ a0025185. 
, and 2013: Decisions with uncertainty: The glass half full. Curr. Dir. Psychol. Sci., 22, 308-315, https://doi.org/ 10.1177/0963721413481473.

, and R. Demnitz, 2019: Communicating climate change: Probabilistic expressions and concrete events. Wea. Climate Soc., 11, 651-664, https://doi.org/10.1175/WCAS-D-18-0126.1. , and J. LeClerc, 2016: Climate projections and uncertainty communication. Top. Cognit. Sci., 8, 222-241, https://doi.org/ 10.1111/tops.12177.

Kahneman, D., and A. Tversky, 1979: Prospect theory: An analysis of decision under risk. Econometrica, 47, 263-291, https:// doi.org/10.2307/1914185.

— substitution in intuitive judgment. Heuristics and Biases: The Psychology of Intuitive Judgment, T. Gilovich, D. Griffin, and D. Kahneman, Eds., Cambridge University Press, 49-81.

, and G. Klein, 2009: Conditions for intuitive expertise: A failure to disagree. Amer. Psychol., 64, 515-526, https:// doi.org/10.1037/a0016755.

, S. P. Slovic, P. Slovic, and A. Tversky, Eds., 1982: Judgment Under Uncertainty: Heuristics and Biases. Cambridge University Press, 555 pp.
Lazo, J. K., A. Bostrom, R. E. Morss, J. L. Demuth, and H. Lazrus, 2015: Factors affecting hurricane evacuation intentions. Risk Anal., 35, 1837-1857, https://doi.org/10.1111/risa.12407.

LeClerc, J., and S. Joslyn, 2015: The cry wolf effect and weatherrelated decision making. Risk Anal., 35, 385-395, https:// doi.org/10.1111/risa.12336.

Lindell, M. K., 2012: Response to environmental disasters. The Oxford Handbook of Environmental and Conservation Psychology, S. D. Clayton, Ed., Oxford University Press, 391-412.

Morss, R. E., J. L. Demuth, and J. K. Lazo, 2008: Communicating uncertainty in weather forecasts: A survey of the U.S. public. Wea. Forecasting, 23, 974-991, https://doi.org/10.1175/ 2008WAF2007088.1.

Tetlock, P. E., 2017: Expert Political Judgment: How Good Is It? How Can We Know?. Princeton University Press, 368 pp.

— of Prediction. Crown Publishers/Random House, 352 pp.

Zhang, F., R. E. Morss, J. A. Sippel, T. K. Beckman, N. C. Clements, N. L. Hampshire, and S. Wang, 2007: An in-person survey investigating public perceptions of and responses to Hurricane Rita forecasts along the Texas coast. Wea. Forecasting, 22 , 1177-1190, https://doi.org/10.1175/2007WAF2006118.1. 